DAMTP-R92/35

September 1992

\title{
Cosmic Strings and Chronology Protection
}

\author{
James D.E. Grant ${ }^{\dagger}$ \\ Department of Applied Mathematics and Theoretical Physics, \\ University of Cambridge, Silver Street, Cambridge CB3 9EW, United Kingdom. \\ Theoretical Astrophysics, Californian Institute of Technology, \\ Pasadena, California 91125.
}

\begin{abstract}
A space consisting of two rapidly moving cosmic strings has recently been constructed by Gott that contains closed timelike curves. The global structure of this space is analysed and is found that, away from the strings, the space is identical to a generalised Misner space. The vacuum expectation value of the energy momentum tensor for a conformally coupled scalar field is calculated on this generalised Misner space. It is found to diverge very weakly on the Chronology horizon, but more strongly on the polarised hypersurfaces. The divergence on the polarised hypersurfaces is strong enough that when the proper geodesic interval around any polarised hypersurface is of order the Planck length squared, the perturbation to the metric caused by the backreaction will be of order one. Thus we expect the structure of the space will be radically altered by the backreaction before quantum gravitational effects become important. This suggests that Hawking's 'Chronology Protection Conjecture' holds for spaces with non-compactly generated Chronology horizon.
\end{abstract}




\section{Introduction}

It has long been known that changes of spatial topology give rise to either singularities or closed timelike curves [1]. It was therefore not too surprising, with hindsight, when several topologically non-trivial spaces were recently constructed that generically contained closed timelike curves [2-4]. These spaces were formed by removing two spheres from a spacetime and then joining the resulting holes together by a cylinder to form a "wormhole". It was found thatif one of the wormhole mouths was in a generic gravitational field, or if the wormhole mouths were in generic relative motion, then closed timelike curves would form. One of the major drawbacks of these spaces, however, is that they are not vacuum solutions of Einstein's equations, and the matter required to maintain the spaces must violate an averaged form of the Weak Energy Condition [3].

A simpler space with closed timelike curves has now been constructed by Gott [5]. Gott's space just contains two cosmic strings moving past each other at high velocity. The space is locally flat away from the strings, so there is no violation of the Weak Energy Condition, and the topology is just $R^{4}$.

The aim of the present paper is to study the quantum mechanical stability of the Gott spacetime. We consider putting a conformally coupled scalar field into the Gott space background and calculate the vacuum expectation value of its energy momentum tensor. Early calculations of such quantities in spaces with closed timelike curves [6] suggested that such quantities would diverge at the Chronology horizon (the boundary of the region containing closed timelike curves). It was then shown in [7] that in any spacetime with closed timelike curves, the Chronology horizon is the limiting surface of a family of polarised hypersurfaces, and that the energy momentum tensor of the field will diverge on all of the polarised hypersurfaces (See also [8] for a specific example). This means that in any space with closed timelike curves there will be surfaces arbitrarily close to the Chronology horizon where the energy momentum tensor is divergent. If this divergent energy momentum tensor is now used as a source term in the semi-classical Einstein equations, $R_{a b}-\frac{1}{2} R g_{a b}=8 \pi l_{P}^{2}\left\langle T_{a b}\right\rangle$, then we expect the backreaction to radically alter the spacetime round about the Chronology horizon, and stop us reaching the causality violating region. 
In Gott's space, we find that the divergence of the energy momentum tensor is very weak at the Chronology horizon. The perturbation of the metric due to the backreaction of this divergence would be unobservable even when we are a Planck length, $l_{P}$, from the Chronology horizon. However, the divergence is stronger as we approach the polarised hypersurfaces. Here we find that when the proper geodesic distance squared around any polarised hypersurface, $\sigma_{n}$ for some integer $n$, is of order $l_{P}^{2}$, then the metric perturbation is of order 1 . This will radically alter the structure of the spacetime, and suggests that Hawking's 'Chronology Protection Conjecture', originally only meant to apply to spaces with compactly generated Chronology horizons, will also apply in the non-compactly generated case.

We begin in section 2 with a brief review of the Gott construction, and section 3 is devoted to an analysis of the space's geometrical properties. The conclusion here is that, far from the strings, the space is identical to a generalised Misner universe, the relevant properties of which are then reviewed. We then calculate, in section 4 , the vacuum expectation value of the energy momentum tensor for a conformally coupled scalar field on this generalised Misner space space. In section 5 we discuss these results and their implications for Hawking's 'Chronology Protection Conjecture'.

\section{Gott's Cosmic String Spacetime}

We consider a space containing an infinitely long, straight cosmic string. This can be viewed as flat Minkowski space with a wedge of angle $2 \alpha$ cut out along the axis of the string. We can choose Minkowski coordinates, $(t, x, y, z)$, and place the core of the string on the line $x=0, y=d$, with $z$ as the coordinate along the axis of the string. We remove the wedge from the space so that points with $x= \pm(y-d) \tan \alpha$ are identified (see fig.1).

Suppose we now consider two points $A$ and $B$ at rest on the surface $y=0$, where $x_{A}^{a}=\left(t, x_{0}, 0,0\right), x_{B}^{a}=\left(t,-x_{0}, 0,0\right)$. There are now two paths that a light signal sent from $A$ could follow to arrive at $B$. The first would be the direct path $A O B$. If $x_{0}$ is big enough, then there is an alternative path, $A C D B$, that goes around the cosmic string, making use of the angular deficit. If $x_{0} \tan \alpha>>d$, then this second light ray will arrive at $B$ before the direct one. 
If a light beam travelling around the cosmic string can arrive before the light beam passing through $O$, then so can a rocket travelling at sufficiently high velocity. The event of the rocket leaving $A, x_{i}$, and arriving at $B, x_{f}$, will be spacelike separated, since the light ray travelling along $y=0$ arrives at $B$ after event $x_{f}$. Hence we can find a Lorentz frame, in which the string moves at velocity $v$ in the $+x$ direction, in which the events $x_{i}$ and $x_{f}$ are simultaneous, i.e. the rocket is seen to arrive at point $B$ at the same time as it left point $A$.

We can take two copies of the above space and glue them together along their $y=0$ surfaces. We boost the region $y \geq 0$ at velocity $v$ in the $+x$ direction, and the region $y \leq 0$ at velocity $v$ in the $-x$ direction. Physically, if we are in the centre of mass frame, all this means is that we see two cosmic strings going in opposite directions at speed $v$, with impact parameter $2 d$ (see fig. 2).

The construction above showed that in the centre of mass frame, we could see a rocket leaving event $x_{i}$ and simultaneously arriving at event $x_{f}$ if it followed the path $A C D B$. If the rocket then turns around at $x_{f}$, then by the same argument it can travel back round the lower cosmic string by path $B E F A$ and arrive back at event $x_{i}$. We have thus created a closed timelike curve through event $x_{i}$.

There is, however, a restriction on the velocity $v$. It can be shown [5] that, if $x_{0}>>d$, then we need

$$
\cosh \xi \sin \alpha>1
$$

in order to get closed timelike curves, where $v=\tanh \xi$. Grand Unified Theories usually predict $\alpha \approx 10^{-5}$, meaning that $v \approx c\left(1-10^{-10}\right)$. This may seem rather unrealistic, but it is possible that cosmic strings created in the early universe would have such high velocities. 


\section{Geometry Of The Space}

Following [9], we now look for a more geometrically transparent representation of the above construction.

If one considers parallel transport of vectors around a closed curve in a spacetime that includes a cosmic string, then there is a non trivial holonomy if the closed curve encloses the string. If the string is at rest, this holonomy is just a rotation through angle $2 \alpha$, where $2 \alpha$ is the deficit angle of the string. If the string is moving at constant velocity $v$ in the positive $x$ direction, then the holonomy is represented by the matrix $B(v) R(2 \alpha) B(-v)$, where $B(v)$ is the boost matrix corresponding to velocity $v$, and $R(2 \alpha)$ is the matrix corresponding to a rotation through angle $2 \alpha$. In the case of the Gott spacetime, the holonomy matrix for a closed curve around both strings will be

$$
H(v, \alpha)=(B(-v) R(2 \alpha) B(v))(B(v) R(2 \alpha) B(-v)) .
$$

We would like to know if this corresponds to a rotation, or a boost. Therefore we consider the trace of $H$, which will be less than 4 if $H$ corresponds to a rotation and greater than 4 if $H$ corresponds to a boost. If we take $\tanh \xi=v$, then we find

$$
\operatorname{Tr}(H)-4=8 \cosh ^{2} \xi(1-\cos 2 \alpha)\left(\sin ^{2} \alpha \cosh ^{2} \xi-1\right) .
$$

The Gott space has closed timelike curves if $\sin \alpha \cosh \xi>1$, and thus corresponds to a holonomy of a boost.

The Gott space is flat away from the strings. The holonomy around a closed curve that encloses both cosmic strings is a boost. This suggests that one could view the region with closed timelike curves as flat Minkowski space identified under the action of a boost.

The exact identification we require can be found by tracing a curve in the Gott space that would usually close up in flat space. This is similar to the case with one cosmic string where a curve that would close up in flat Minkowski space will not close up if the curve goes around the string. The amount the curve does not close up by is a rotation about the axis of the string, through the deficit angle of the string. Therefore in this case, the amount that the curve does not close up by is the same as the holonomy around the string. 
In general the endpoint of the curve will be $x^{\prime}=H x+C$, where $H$ is the holonomy around any closed curve enclosing the string, and $C$ is some constant vector.

Defining the function

$$
\Delta=\cosh ^{2} \xi \sin ^{2} \alpha-1,
$$

and expressing the results in terms of the coordinates

$$
\begin{gathered}
t^{\prime}=\frac{1}{\Delta}[t \sinh \xi \sin \alpha-y \cos \alpha]-\frac{d}{2} \cosh ^{2} \xi \sin 2 \alpha\left(4 \sin ^{2} \alpha+(2 \Delta+1)^{2}\right), \\
x^{\prime}=x-\frac{d}{\Delta} \cosh \xi \sin \alpha \sin 2 \alpha(2 \Delta+1)\left(1+\cosh ^{2} \xi \Delta\right), \\
y^{\prime}=\frac{1}{\Delta}[y \sinh \xi \sin \alpha-t \cos \alpha],
\end{gathered}
$$

it is straightforward to show that the components of $H$ and $C$ are given by

$$
\begin{gathered}
H(\alpha, \xi)=\left(\begin{array}{cccc}
1+f(\alpha, \xi) \Delta & g(\alpha, \xi) \Delta & 0 & 0 \\
g(\alpha, \xi) & 1+f(\alpha, \xi) \Delta & 0 & 0 \\
0 & 0 & 1 & 0 \\
0 & 0 & 0 & 1
\end{array}\right), \\
C^{y^{\prime}}=-\frac{4 d}{\Delta} \sinh \xi \sin \alpha, \\
C^{t^{\prime}}=C^{x^{\prime}}=C^{z}=0 .
\end{gathered}
$$

Here we have defined the functions

$$
\begin{gathered}
f(\alpha, \xi)=4 \cosh ^{2} \xi(1-\cos 2 \alpha), \\
g(\alpha, \xi)=4 \cosh \xi \sin \alpha\left(\cosh ^{2} \xi(1-\cos 2 \alpha)-1\right) .
\end{gathered}
$$


If we assume there are closed timelike curves, then (2.1) implies that $\Delta>0$. If we change to coordinates $\tilde{t}=\Delta^{1 / 2} t^{\prime}, \tilde{y}=\Delta^{1 / 2} y^{\prime}$, the metric becomes the flat space metric $d s^{2}=-d \tilde{t}^{2}+d x^{\prime 2}+d \tilde{y}^{2}+d z^{2}$. In terms of these coordinates, the holonomy takes the form of a boost in the $\tilde{t}-x^{\prime}$ plane with parameter $a$ given by

$$
\cosh a=1+f(\alpha, \xi) \Delta,
$$

and the vector $C$ becomes a displacement in the $\tilde{y}$ direction of distance $b$, where

$$
b=-\frac{4 d}{\Delta^{1 / 2}} \sinh \xi \sin \alpha .
$$

Thus, for any observer that travels around both strings, the Gott space will be physically indistinguishable from flat space identified under the combined discrete action of a boost in the $\tilde{t}-x^{\prime}$ plane and a translation in the $\tilde{y}$ plane. (From now on we drop the tildes and primes on these coordinates.)

This is just a generalisation of Misner space [10,11]. Here we pick an origin, $O$, in flat 2 dimensional Minkowski space, and identify the points $A^{n}(x)$, for all integers $n$ and $x \in J^{-}(O)$, where

$$
A^{n}(x) \equiv(t \cosh n a+x \sinh n a, x \cosh n a+t \sinh n a) .
$$

Under a Lorentz boost of velocity $v=\tanh a$, the point $x$ is carried to the point $A(x)$. Thus, physically, Misner space corresponds to the bottom quadrant of Minkowski space identified under the action of a discrete boost.

Introducing coordinates $T$ and $X$ such that $t=-T \cosh X, x=-T \sinh X$, the metric becomes $d s^{2}=-d T^{2}+T^{2} d X^{2}$ and the above identified region corresponds to $T>0$ with coordinate $X$ having period $a$. We can extend this metric through the surface $T=0$, where it becomes degenerate, by introducing coordinates $\tau=T^{2}, u=X-\log T$, giving the metric $d s^{2}=d u d \tau+\tau d u^{2}$, which is non-degenerate for all real $\tau$. The region $\tau<0$ now contains closed timelike curves, and the surface $\tau=0$ contains closed null geodesics. This extended space corresponds to the bottom and left hand wedges of Minkowski space identified under the action of the boost defined by $A$ above. One can do similar extensions 
and consider the whole of the two dimensional Minkowski space being identified under this discrete boost. In order for the resulting space to be a manifold, however, we must delete the origin, $O$. The resulting manifold is then non-Hausdorff and the space is geodesically incomplete [11].

Two flat dimensions can now be added to the above space. We then have the freedom to make identifications in these extra directions. The discussion above suggests that the Gott space is the same as four dimensional Minkowski space with the points $B^{n}(x)$ identified, for all integers $n$, where

$$
B^{n}(x) \equiv(t \cosh n a+x \sinh n a, x \cosh n a+t \sinh n a, y+n b, z),
$$

and $a$ and $b$ are defined by (3.12) and (3.13) respectively. As long as $b \neq 0$, no points need be removed from the space. The resulting manifold is Hausdorff, and tghe space is geodesically complete. The fact that the Gott space has closed timelike curves at any value of $t$ [12] is now analagous to the fact that the identified left (and right) hand quadrant of identified Minkowski space have closed timelike curves at arbitrary values of the Minkowski coordinate $t$. Further, the fact that in Misner space the surfaces $\tau=$ constant $>0$ are not intersected by any closed timelike curves suggests that there will exist similar achronal surfaces in the Gott space which are not on any closed timelike curves [13].

Any point in the covering Minkowski space is null separated from another copy of the same point if its coordinates satisfy

$$
x^{2}-t^{2}=\frac{n^{2} b^{2}}{2(\cosh n a-1)} .
$$

Thus every point on this surface can be joined to itself by a (unique) null geodesic that passes around both strings $n$ times. Although, in the physical space, the above null geodesic passes through the same point twice, its tangent vector differs on these two occasions by the holonomy $H^{n}$. Following [13] and [7] respectively, we call such lines self intersecting null geodesics and call the surface defined by (3.16) the $n$ 'th polarised hypersurface. We reserve the term closed null geodesic for a line whose tangent vector coincides at the point each time, and thus goes through the point an infinite number of times. 
If we take the limit $n \rightarrow \infty$ in (3.16) then we find that the chronology horizon is situated at $t= \pm x$. This is a null surface, but unlike in the ordinary Misner space case it does not contain any closed null geodesics. This is because any null geodesic in the surface must have $y=$ constant, and so cannot join two identified points. Therefore the null geodesics that generate the horizon will never enter and remain within a compact region when followed backwards in time. So, in the terminology of [14] the Chronology horizon is non-compactly generated.

\section{Matter Fields On The Space}

We here use the results of the preceding sections to consider placing quantum mechanical matter into the Gott space. For simplicity we take a conformally coupled scalar field, and we calculate the vacuum expectation value of the energy momentum tensor for this field, $\left\langle T_{a b}\right\rangle$. In a flat four dimensional space, the renormalised propagator of a scalar field is

$$
G\left(x, x^{\prime}\right)=\frac{1}{(2 \pi)^{2}} \sum_{\substack{n=-\infty \\ n \neq 0}}^{+\infty} \sigma_{n}\left(x, x^{\prime}\right)^{-1}
$$

where $\sigma_{n}\left(x, x^{\prime}\right)$ is the square of the proper geodesic distance from $x$ to $x^{\prime}$ along the $n$ 'th geodesic joining the two points. To calculate $\left\langle T_{a b}\right\rangle$, we differentiate this propagator twice with respect to position and take the limit $x^{\prime} \rightarrow x$ (see (4.5)). Thus we would expect $\left\langle T_{a b}(x)\right\rangle$ to behave like

$$
\left\langle T_{a b}(x)\right\rangle \sim \lim _{x^{\prime} \rightarrow x} \sum_{\substack{n=-\infty \\ n \neq 0}}^{+\infty} \sigma_{n}\left(x, x^{\prime}\right)^{-3} .
$$

If there is a self intersecting null geodesic through $x$, then one of the $\sigma_{n}(x, x)$ will vanish, and so $\left\langle T_{a b}\right\rangle$ will diverge at $x$. If one now makes a semi-classical approximation and treats $\left\langle T_{a b}\right\rangle$ as a source term in the Einstein field equations, one might hope that the divergence of $\left\langle T_{a b}\right\rangle$ on the polarised hypersurfaces would induce a singularity, making these surfaces non-traversable. Since there are polarised hypersurfaces arbitrarily close to the 
Chronology horizon, we would therefore hope that these divergences would also make the Chronology horizon non-traversable. This is the basis upon which Hawking put forward the 'Chronology Protection Conjecture' which states that closed timelike curves cannot be created [14].

In the covering space of Gott space, we begin with the ordinary flat space propagator

$$
G_{0}\left(x, x^{\prime}\right)=\frac{1}{(2 \pi)^{2}}\left[-\left(t-t^{\prime}\right)^{2}+\left(x-x^{\prime}\right)^{2}+\left(y-y^{\prime}\right)^{2}+\left(z-z^{\prime}\right)^{2}\right]^{-1}
$$

The renormalised propagator on the identified spacetime is then

$$
\begin{gathered}
G\left(x, x^{\prime}\right)=\frac{1}{(2 \pi)^{2}} \sum_{\substack{n=-\infty \\
n \neq 0}}^{+\infty}\left[-\left(t-\left(t^{\prime} \cosh n a+x^{\prime} \sinh n a\right)\right)^{2}+\left(x-\left(x^{\prime} \cosh n a+t^{\prime} \sinh n a\right)\right)^{2}\right. \\
\left.+\left(y-\left(y^{\prime}+n b\right)\right)^{2}+\left(z-z^{\prime}\right)^{2}\right]^{-1}
\end{gathered}
$$

This propagator is already symmetric under interchange of $x$ and $x^{\prime}$, so we obtain the renormalised energy momentum tensor of the field [15] from

$$
\left\langle T_{a b}\right\rangle=\lim _{x^{\prime} \rightarrow x}\left[\frac{2}{3} \nabla_{a} \nabla_{b^{\prime}}-\frac{1}{3} \nabla_{a} \nabla_{b}-\frac{1}{6} g_{a b} \nabla_{c^{\prime}} \nabla^{c^{\prime}}\right] G\left(x, x^{\prime}\right) .
$$

On carrying out this calculation with the above propagator, one finds that the only non-zero components of $\left\langle T^{a}{ }_{b}\right\rangle$ are

$$
\begin{gathered}
\left\langle T_{T}^{T}\right\rangle=\frac{1}{3 \pi^{2}} \sum_{n=1}^{\infty} \frac{(\cosh n a+2)}{f_{n}^{2}} \\
\left\langle T_{X}^{X}\right\rangle=\frac{1}{3 \pi^{2}} \sum_{n=1}^{\infty} \frac{(\cosh n a+2)}{f_{n}^{2}}\left[-3+\frac{4 n^{2} b^{2}}{f_{n}}\right], \\
\left\langle T_{y}^{y}\right\rangle=\frac{1}{3 \pi^{2}} \sum_{n=1}^{\infty}\left[\frac{(\cosh n a+2)}{f_{n}^{2}}-\frac{2 n^{2} b^{2}(\cosh n a+5)}{f_{n}^{3}}\right],
\end{gathered}
$$




$$
\left\langle T_{z}^{z}\right\rangle=\frac{1}{3 \pi^{2}} \sum_{n=1}^{\infty}\left[\frac{(\cosh n a+2)}{f_{n}^{2}}-\frac{2 n^{2} b^{2}(\cosh n a-1)}{f_{n}^{3}}\right],
$$

where $t=T \cosh X, x=T \sinh X$ and

$$
f_{n}=2\left(t^{2}-x^{2}\right)(\cosh n a-1)+n^{2} b^{2}=2 T^{2}(\cosh n a-1)+n^{2} b^{2} .
$$

These expressions diverge on the Chronology horizon, where $T=0$, and on the polarised hypersurfaces, where $f_{n}=0$ for some integer $n$. (We note in passing that the ( $T, X, y, z)$ coordinate system becomes singular at $T=0$, the Chronology horizon.)

If we approach the Chronology horizon, we can approximate the above sums by integrals, and evaluate the asymptotic behaviour by a saddle point method. We find that the components of the energy momentum tensor diverge like $K / b^{2} T^{2}$, where $K$ is a negative constant. We can estimate the perturbation this will cause in the metric by using it as a source term in the semi-classical Einstein equations, $R_{a b}-\frac{1}{2} R g_{a b}=8 \pi l_{P}^{2}\left\langle T_{a b}\right\rangle$, where $l_{P}$ is the Planck length. Therefore the perturbation to the curvature will be of order $K l_{P}^{2} / b^{2} T^{2}[16]$. To find the metric perturbation felt by someone travelling along a geodesic $(X, y, z)=$ constant, we have to integrate twice with respect to $T$, giving

$$
\delta g \approx \frac{K l_{p}^{2}}{b^{2}} \log (T / b)
$$

This perturbation diverges at the Chronology horizon. However, we expect quantum gravity will come into play before the horizon, and may smooth out the divergence before it becomes noticeable. It seems reasonable to assume that quantum gravitational effects will become important at some Lorentz invariant, observer independent distance from the horizon [14]. Thus a first approximation would be that quantum gravitational effects come into play when

$$
T \approx l_{P}
$$

Putting this into (4.8), and assuming that $b$ is some typical macroscopic distance of 
order one metre, gives a metric perturbation of order $10^{-70}$. This would be completely unobservable.

We can study the behaviour of $\left\langle T^{a}{ }_{b}\right\rangle$ near the polarised hypersurfaces by defining a coordinate $\tilde{T}$ by $\tilde{T}^{2}=x^{2}-t^{2}$. We find that the components of $\left\langle T^{a}{ }_{b}\right\rangle$ diverge, at worst, like $K^{\prime} b^{2} /\left(\tilde{T}+\tilde{T}_{n}\right)^{3}\left(\tilde{T}-\tilde{T}_{n}\right)^{3}$ as we approach the $n$ 'th polarised hypersurface, where $\tilde{T}_{n}$ is the value of $\tilde{T}$ on that surface, and $K^{\prime}$ is a constant. This means that the dominant contribution to the metric perturbation caused by the back reaction of the matter will be

$$
\delta g_{n} \approx \frac{K^{\prime} l_{p}^{2} b^{2}}{\left(\tilde{T}+\tilde{T}_{n}\right)^{3}\left(\tilde{T}-\tilde{T}_{n}\right)}
$$

It is more difficult to estimate when quantum gravitational effects become strong close to the the polarised hypersurfaces. If we were to treat the gravitational field like a massless spin 2 field in flat spacetime, we would expect the quantum fluctuations of the field to be governed by the geodetic interval around the polarised hypersurface $\sigma_{n}(x, x)[17]$. This suggests that quantum gravity would become significant when $\left(\tilde{T}+\tilde{T}_{n}\right)\left(\tilde{T}_{-}-\tilde{T}_{n}\right) \approx l_{P}^{2}$. This leads to a metric perturbation of order 1 , which would radically alter the structure of the space around the polarised hypersurfaces and the Chronology horizon ${ }^{1}$.

Thus, it appears that around the polarised hypersurfaces, quantum gravity will not enter until the metric perturbation has become large enough to change the structure of the space.

\footnotetext{
1 I am grateful to Kip Thorne for this argument; see [18].
} 


\section{Conclusion}

We have shown that, away from the strings, the Gott space is identical to flat Minkowski space identified under the action of a discrete boost and translation. On calculating the vacuum expectation value of the energy momentum tensor for a conformally coupled scalar field on this space, we find that it diverges on the Chronology horizon and on the polarised hypersurfaces. The divergence around the polarised hypersurfaces is sufficiently strong that we expect the backreaction of the field to radically alter the structure of the space before quantum gravitational effects have come into play.

These results seem to extend Hawking's 'Chronology Protection Conjecture' which states that closed timelike curves cannot be created [14]. The Chronology Protection Conjecture originally only refered to spaces where the region of closed timelike curves was compact, but our results seem to suggest that it also applies in spaces with non-compactly generated Chronology horizons.

It remains to be shown that the Green function of the wave equation given by (4.4) corresponds to the propagator of a physically acceptable quantum state of the field on the space (in the sense of [19]). Indeed, there are serious problems in doing quantum field theory on any non-globally hyperbolic spacetime (see [20] for one approach to this problem). It has been shown, however, that the Green function constructed in [6] for Misner space is a propagator for a real quantum state and that the Hiscock-Konkowski state is actually a thermal state [21]. Hopefully, similar arguments should apply to the present case. 


\section{Acknowledgements}

I would like to thank Stephen Hawking and Kip Thorne for their guidance and suggestions. I also wish to convey my thanks to the Theoretical Astrophysics group in Caltech, who were so helpful and encouraging when this work was being completed there. I also must thank St. John's College, Cambridge, for a travel grant which enabled me to visit Caltech.

This research was carried out under a Science and Engineering Research Council (SERC) studentship.

$\dagger \quad$ email address : jdeg1@phx.cam.ac.uk.

[1] R. P. Geroch, J. Math. Phys 8, 782 (1967).

[2] M. S. Morris and K. S. Thorne, Am. J. Phys. 56, 395 (1988).

[3] M. S. Morris, K. S. Thorne and U. Yurtsever, Phys. Rev. Lett. 61, 1446 (1988).

[4] V. P. Frolov and I. D. Novikov, Phys. Rev. D42, 1057 (1990).

[5] J. R. Gott, Phys. Rev. Lett. 66, 1126 (1991).

[6] W. Hiscock \& D. A. Konkowski, Phys. Rev. D26, 1225 (1982).

[7] S.-W. Kim and K. S. Thorne, Phys. Rev. D43, 3929 (1991).

[8] V. P. Frolov, Phys. Rev. D43, 3878 (1991).

[9] S. Deser, R. Jackiw and G. t'Hooft, Annals of Physics, 152, 220 (1984).

[10] C. W. Misner in Relativity Theory and Astrophysics I: Relativity and Cosmology, edited by J. Ehlers, Lectures in Applied Mathematics, Volume 8 (American Mathematical Society, 1967).

[11] The Large Scale Structure of Space-time, S. W. Hawking \& G. F. R. Ellis (Cambridge University Press, Cambridge, 1973).

[12] A. Ori, Phys. Rev. D44, 2214 (1992).

[13] C. Cutler, Phys. Rev. D45, 487 (1992).

[14] S. W. Hawking, 'The Chronology Protection Conjecture', DAMTP preprint R91/15, submitted to Phys. Rev. D.

[15] R. M. Wald, Phys. Rev. D17, 1477 (1978). 
[16] Kip Thorne (private communication) has done similar calculations with the energy momentum tensor in [7] for the wormhole spacetime. Here the Chronology horizon is compactly generated and thus contains a smooth closed null geodesic. He finds that if the wormhole has no defocusing effect $(b / 2 D=1$ in the terminology of [7]), then $\left\langle T^{a b}\right\rangle$ diverges like the inverse square of time $T$ to the horizon (note: this $T$ is analagous to the coordinate $t$ in our terminology). Thus the divergence in this case is similar to that in our generalised Misner space. However, if the defocusing is non-zero $(b / 2 D \neq 1)$ then the divergence is weaker and if $b / 2 D$ is sufficiently small, there is no divergence on the Chronology horizon except on the closed null geodesic. This may be the generic behaviour away from the closed null geodesic on a space where the Chronology horizon is compactly generated.

[17] It may be helpful to say what we mean by $\sigma_{n}(x, x)$, when $x$ is not on the $n$ 'th polarised hypersurface. We can look on $x$ as the first of an infinite set of points, $\left\{x_{i}: i=\right.$ $\left.1,2,3 \ldots: x_{1}=x\right\}$, that converge to some point $y$ on the $n$ 'th polarised hypersurface. Since $y$ is on the $n$ 'th polarised hypersurface, there will be a self intersecting null geodesic, $\mathcal{G}$, through $y$. This self intersecting null geodesic is the limit of a set of closed spacelike geodesics, $\mathcal{G}_{i}$, through the corresponding points $x_{i} . \sigma_{n}(x, x)$ is then the proper geodesic interval around the closed spacelike geodesic $\mathcal{G}_{1}$. For this reason, we refer to $\sigma_{n}(x, x)$ as the proper geodesic interval around the polarised hypersurface.

[18] K. S. Thorne in Proceedings of 13th International Conference on General Relativity and Gravitation, ed. C. Kozameh (Institute of Physics, Bristol, England, in press).

[19] B. S. Kay in Differential Geometrical Methods in Theoretical Physics, eds. K. Bleuler and M. Werner (Kluwer Academic Publishers, 1988).

[20] B. S. Kay, 'The Principle of Locality and Quantum Field Theory on (Non-Globally Hyperbolic) Curved Spacetimes', DAMTP preprint R92/22, to be published in Rev. Theor. Phys.

[21] B. S. Kay, unpublished. 